\title{
Testing the prenatal androgen hypothesis: measuring digit ratios, sexual orientation, and spatial abilities in adults
}

\author{
Sari M. van Anders, Elizabeth Hampson* \\ Department of Psychology, University of Western Ontario, London, ON, Canada
}

Received 17 June 2004; revised 1 September 2004; accepted 13 September 2004

Available online 22 October 2004

\begin{abstract}
The present study examined whether the following variables putatively associated with prenatal androgens are inter-related in women: spatial abilities, sexual orientation, and 2nd to 4th finger (digit) length ratio (2D:4D). Participants were 99 healthy premenopausal women tested in the menstrual phase of the ovarian cycle between 0800 and $0930 \mathrm{hr}$. Women completed the Kinsey scales of sexual orientation, and were either strictly heterosexual (HS; $N=79$ ) or not-strictly heterosexual (NHS; $N=20$ ). Photocopies of the two hands were collected, and participants completed the revised Vandenberg Mental Rotations test, the Paper Folding test, and a short version of the Guilford-Zimmerman Spatial Orientation Test. Results showed that NHS women exhibited superior spatial ability relative to HS women. No significant difference was found between the HS and NHS women in the 2D:4D digit ratio. There was no association between the digit ratio and spatial performance. These results support an association between increased spatial abilities and heteroflexible sexual orientation, which may possibly be mediated by high prenatal androgens.
\end{abstract}

(C) 2004 Elsevier Inc. All rights reserved.

Keywords: Prenatal androgen; Sexual orientation; Digit ratio; Spatial abilities; Mental rotation; Paper folding; Spatial navigation; 2D:4D

There are various physiological markers in humans that are sexually dimorphic. One is the ratio of the second digit to fourth digit (2D:4D) on the hand, which is significantly higher in women than in men (Manning et al., 1998, 2000; Peters et al., 2002; Lippa, 2003). Manning et al. (1998) found that the sex difference was visible in two-year olds (the youngest group sampled), and suggested that the ratios were probably established in utero. This sex difference is thought to be due to differences in prenatal androgen exposure, and studies of individuals with congenital adrenal hyperplasia (CAH), a disorder in which the fetus is exposed to excess androgens, have provided some support. For example, two studies have shown that females with $\mathrm{CAH}$ exhibit lower digit ratios than female controls (Brown et al.,

* Corresponding author. Department of Psychology, University of Western Ontario, London, ON N6A 5C2 Canada. Fax: +1 5196613213.

E-mail address: ehampson@uwo.ca (E. Hampson). 2002b; Ökten et al., 2002). One other study, however, failed to find this difference (Buck et al., 2003).

High prenatal androgens have been associated with an increase in 'heteroflexible' (i.e. non-strictly heterosexual) or lesbian orientation in women. For example, women with $\mathrm{CAH}$ exhibit lower rates of exclusively heterosexual fantasy than unaffected female relatives (Zucker et al., 1996) and higher rates of bisexual or homosexual fantasy or experience (Dittmann et al., 1992; Ehrhardt et al., 1968; Money et al., 1984). Conversely, a heteroflexible or lesbian orientation has been associated with putative markers of high prenatal androgens. Click-evoked otoacoustic emissions are believed to be sensitive to prenatal androgens (McFadden, 1998), and lesbians and bisexual women show a pattern that is intermediate to heterosexual men and women (McFadden and Champlin, 2000; McFadden and Pasanen, 1998).

A lesbian orientation has also been linked with high androgens in women using the 2D:4D marker. Lesbians exhibit smaller digit ratios than heterosexual women 
(McFadden and Shubel, 2002; Rahman and Wilson, 2003b; Williams et al., 2000; c.f. Lippa, 2003) and self-identified butch lesbians have smaller ratios than femme lesbians (Brown et al., 2002a). In a study of monozygotic twin pairs, Hall and Love (2003) found that lesbian co-twins had significantly lower ratios than their heterosexual co-twins, suggesting a non-genetic contribution to both sexual orientation and 2D:4D.

Spatial ability is another characteristic that has been associated with prenatal androgens. Men outscore women on tests of mental rotation and spatial perception (Voyer et al., 1995). Part of the sex difference seems to be attributable to circulating steroids at the time of testing (Hampson, 1990a, 1990b; Hausmann et al., 2000), but studies of girls with $\mathrm{CAH}$ suggest there is an additional, organizational, component. For example, CAH girls outperformed controls on tests of spatial ability, but not other tests (Hampson et al., 1998; Resnick et al., 1986). Helleday et al. (1994) did not find superior spatial ability in $\mathrm{CAH}$ women compared to controls, but the groups were not matched in overall intelligence, rendering a comparison of abilities difficult. Although research on $\mathrm{CAH}$ has produced inconsistent findings (Hines et al., 2003), Grimshaw et al. (1995) found that androgen concentrations in amniotic fluid were significantly and positively correlated with subsequent mental rotation ability in girls.

Based on the hypothesized common link of prenatal androgens between 2D:4D, sexual orientation, and spatial abilities, some research has examined inter-correlations among these markers. Two studies found evidence for a link between low digit ratios (i.e. male-typical) and superior spatial performance in women (Csathó et al., 2003) and men (Manning and Taylor, 2001). Another study found a significant negative correlation between 2D:4D on the right hand and mental rotations performance in men, but not women (McFadden and Shubel, 2003). Finally, two studies failed to find a significant association between 2D:4D and mental rotations performance in men or women (Austin et al., 2002; Coolican and Peters, 2003).

An association between a lesbian orientation and higher mental rotations ability has been hypothesized but is controversial. In a large sample of lesbians and heterosexual women, a significant difference in favor of lesbians was seen (Rahman and Wilson, 2003a), while two other studies failed to find significant differences though means were in the expected directions (Neave et al., 1999; Wegesin, 1998). Earlier studies found no significant differences between lesbians and heterosexual women, even with large samples (Gladue and Bailey, 1995; Tuttle and Pillard, 1991).

It is unclear why the results are conflicting, but better control of extraneous variables known to affect spatial performance in women (e.g. phase of the menstrual cycle; time of day, Moffat and Hampson, 1996), and use of large sample sizes, might reduce error variation and allow underlying relationships to be more clearly evaluated.
Previous literature has found associations between high prenatal androgens and low digit ratios, lesbian or heteroflexible orientation, and increased spatial ability. To the extent that these variables are associated with high prenatal androgen exposure at the same point in gestation, we would expect significant correlations between them to exist. The purpose of the present study was to investigate whether individual differences in spatial ability, lesbian or heteroflexible orientation, and in digit ratios were associated, as would be predicted by the prenatal androgen hypothesis.

\section{Materials and methods}

\section{Participants}

Participants were 99 healthy premenopausal women (mean age $=23.76 \mathrm{yrs} ; \mathrm{SD}=5.66$, range $=18-42 \mathrm{yrs}$ ), who were neither pregnant nor breastfeeding, and had not used oral contraceptives for at least 4 months. Participants were classified as strictly heterosexual (HS, $n=79$ ) or nonstrictly heterosexual (NHS, $n=20$ ) (see below for details), but were not recruited based on orientation. There were no significant differences between the HS and NHS women in age ( $M=23.27$ vs. $25.89 \mathrm{yrs})$, weight $(M=63.81$ vs. 63.68 $\mathrm{kg})$, height $(M=166.29$ vs. $167.28 \mathrm{~cm})$, or body mass index $(M=23.08$ vs. 22.87$)$. Participants were recruited through posters and newspaper advertisements at the University of Western Ontario. A reimbursement was provided for participation.

\section{Procedure}

Sessions began at $0800 \mathrm{hr}$ and lasted approximately 1.5 hrs. This controlled for variations in cognitive performance associated with time of day. Women have been shown to perform better on spatial tests in early morning (Moffat and Hampson, 1996). For each woman, testing took place in the first seven days of the menstrual cycle when estrogen levels are lowest. This allowed us to minimize and control effects of estrogen on spatial performance (Hampson, 1990a; 1990b; Hausmann et al., 2000). A specimen of saliva was collected at the beginning and end of the test session to verify that there were no group differences in circulating testosterone that could account for differences in spatial ability.

\section{Digit ratios}

Photocopies of the two hands were made for later calculation of the 2D:4D ratio. Second copies were made if the landmarks used for measuring were lacking in quality. Despite this, digit ratios could not be measured in five women because landmarks could not be clearly identified. The lower landmark was the most basal crease on each finger and the upper landmark was the most distal point on 
the finger tip. Distance between the landmarks was measured twice with a ruler to the nearest $0.5 \mathrm{~mm}$. The digit ratio was calculated for each hand by dividing the length of the second by that of the fourth digit.

The interrater reliability between two blind raters was calculated. Reliabilities for the digit ratios (intra-class correlation coefficients) were: right hand $r_{1}=.94$; left hand $r_{1}=.92$. All ratios reported are the average of the two independent raters.

\section{Sexual orientation}

Participants completed the Kinsey Scale (Kinsey et al., 1948) regarding their sexual fantasies and behaviours. Sexual orientation was rated on a continuum ranging from exclusively heterosexual (0) to exclusively homosexual (6). The women were classified as strictly heterosexual (HS) if they scored zero or one on both measures (Hall and Love, 2003; Rahman and Wilson, 2003b) or as not strictly heterosexual (NHS) if they scored two or more on any measure.

\section{Cognitive tests}

The Paper Folding test (Ekstrom et al., 1976) consists of two parts, each of which has 10 items. Three minutes were allowed to complete each part. Each item shows a paper being folded before a hole is punched through it. The participant selects one of five alternatives that represents the position of the holes in the paper after it has been unfolded. The score was the number correct, adjusted for guessing. Voyer et al. (1995) report that the typical effect size of the sex difference on Paper Folding is about 0.12.

The revised version of the Vandenberg Mental Rotations Test (MRT) (Peters et al., 1995) was used. The test consisted of two parts with $4 \mathrm{~min}$ allowed for each part; each part has 12 items. Each item consisted of a target image of a threedimensional object made of cubes. The participant was instructed to select the two correctly rotated images from four possibilities. The test was scored using the criteria set forth by Vandenberg and Kuse (1978). The size of the sex difference typically found using this method of scoring is 0.70 to 0.94 (Voyer et al., 1995).

A short version of the Guilford-Zimmerman Spatial Orientation test (GZSO) (Guilford and Zimmerman, 1956) was used. The test consisted of 36 questions, with $5 \mathrm{~min}$ allowed for completion. Each question shows the prow of a boat and a distant shoreline with varying details (such as trees, etc.). A second image shows a change in the location of the prow relative to the shoreline, such that the boat may have moved forward, backward, right, left, or may have rolled. Participants must choose which of five possible options represents the change. To correct for guessing, the score was the number of correct answers minus one-quarter of the number incorrect. The test norms for the GZSO show an effect size of approximately 0.80 , which we have corroborated in previous research using the short version (Moffat et al., 1998).

A spatial composite score was calculated for each participant based on their performance on the three spatial tests. Scores on each test were transformed into z-scores, then averaged to provide an unweighted spatial composite zscore.

As a control task, participants were given the Verbal Meaning sub-scale of the Primary Mental Abilities battery (Thurstone, 1962). The scale consists of 60 items, with 4 min allowed for completion. Participants must select one of five words that best approximates the meaning of each target word. The score was the number correct. This test was given to ensure there were no group differences in general ability. Scores on vocabulary tests correlate positively with general IQ (Vernon, 1971; Weschler, 1981), and do not show sex differences.

\section{Saliva collection}

Participants were asked to refrain from eating, drinking (except water), smoking, or brushing their teeth for one hour prior to saliva collection to ensure optimal quality of the saliva. Prior to the first saliva sample, participants rinsed their mouths with water, and saliva production was stimulated with the use of an inert sugar-free gum. The saliva was collected in a polystyrene culture tube pre-treated with sodium azide. Following collection, the tubes were stored at room temperature for 18-24 hrs to allow separation to occur. The tubes were then frozen at $-20^{\circ} \mathrm{C}$ until analysis.

\section{Radioimmunoassays}

Specimens were thawed at $4^{\circ} \mathrm{C}$ and centrifuged at 3000 rpm for $15 \mathrm{~min}$. A double ether extraction was then performed. Due to the large number of specimens, the assay was done in two batches, each using a Coat-a-Count kit for total testosterone (Diagnostic Products, Los Angeles, CA) modified for use with saliva. All specimens were assayed in duplicate. The sensitivity was $2.5 \mathrm{pg} / \mathrm{mL}$ for each assay, and the intra-assay coefficients of variation were $4 \%$ and $9 \%$, respectively. The antiserum used is highly specific to testosterone with negligible cross-reactivity with other steroids except dihydrotestosterone $(<3.5 \%)$. The mean testosterone concentration, averaged across the two duplicates, was used for statistical analysis.

\section{Results}

The purpose of this study was to explore the degree of intercorrelation among three variables, all of which have been linked theoretically to the level of prenatal testosterone exposure. For the analyses involving spatial ability, it was necessary to exclude women using 
psychoactive medications or who had a psychiatric or neurological condition $(n=14)$, as these can adversely affect cognitive function.

\section{Sexual orientation and spatial abilities}

Consistent with the androgen hypothesis, NHS women exhibited better spatial performance than HS women, and this was significant or near significant on all 3 spatial tests: spatial composite, $t(83)=-2.46, p=.016$; Paper Folding, $t(83)=-2.085, p=.040 ;$ MRT, $t(83)=-1.916, p=.059$; GZSO, $t(83)=-1.87, p=.066$. (See Fig. 1 for means). NHS women also exhibited higher scores than HS women on the vocabulary test, $t(83)=-2.10, p=.043$, suggesting a superiority in general intellectual ability. When vocabulary scores were entered as a covariate in analyses of covariance on the spatial measures, NHS women still exhibited better
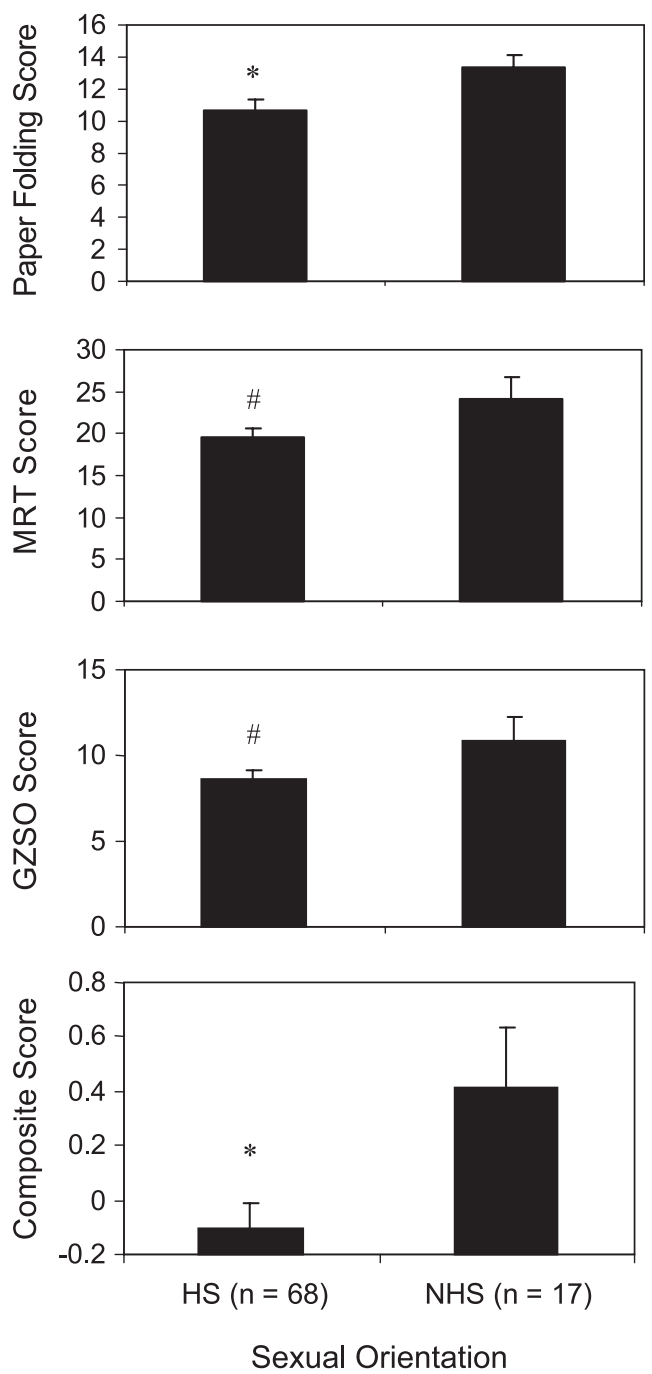

Fig. 1. Mean scores on the Paper Folding Test, Mental Rotations Test (MRT), Guilford-Zimmerman Spatial Orientation Test (GZSO), and spatial composite $z$-score (Composite Score) in strictly heterosexual women (HS) and non-strictly heterosexual women (NHS). Group differences were tested using independent $t$-tests. '*' $p<.05$, '\#’ $p<.10$. spatial performance than HS women. In no case was the covariate significant, resulting in no significant adjustment to the group differences in spatial ability.

To analyse the strength of the association between sexual orientation and spatial ability, the sexual experience and sexual fantasy items from the Kinsey scale were used to compute correlations. Significant positive correlations were seen between same-sex sexual fantasies and spatial performance: spatial composite, Pearson's $r(83)=.29, p=.007$; Paper Folding, $r(83)=.21, p=.053$; MRT, $r(83)=.20, p=$ .070 ; GZSO, $r(83)=.29, p=.007$. There were no significant correlations between same-sex experience and spatial performance, reflecting the restriction of range on this item.

Because circulating testosterone levels may affect spatial performance in women (Moffat and Hampson, 1996), group differences in salivary testosterone were analysed to determine whether differences in circulating testosterone might account for the superior spatial performance in NHS women. However, there was no significant difference between HS $(M=20.34 \mathrm{pg} / \mathrm{mL}, S D=6.13)$ and NHS $(M=21.86 \mathrm{pg} / \mathrm{mL}, S D=6.20)$ women in testosterone levels, $t(82)=-.91, p=.364$.

There were no significant correlations between testosterone and spatial ability in HS women: spatial composite, Pearson's $r(65)=-.01, p=.915$; Paper Folding, $r(65)=.05$, $p=.692$; MRT, $r(65)=-.03, p=.792$; GZSO, $r(65)=-.05$, $p=.669$; nor were there any significant correlations in NHS women: spatial composite, $r(15)=-.08, p=.753$; Paper Folding, $r(15)=.17, p=.515$; MRT, $r(15)=-.09, p=.736$; GZSO, $r(15)=-.22, p=.394$.

\section{Sexual orientation and digit ratios}

No significant group differences were found between HS and NHS women in the 2D:4D digit ratio: right hand ratio (HS $M=.97$; NHS $M=.97$ ), $t(94)=-0.17, p=$ .865 ; left hand ratio (HS $M=.97$; NHS $M=.98), t(95)=$ $-1.17, p=.247$. Digit ratios were not correlated with ratings of either fantasy (RH $r(94)=.01$, LH $r(95)=.02$ ) or same-sex experience (RH $r(94)=.03$, LH $r(95)=$ $-.08)$ in the group as a whole. Investigating the correlations in NHS women only $(n=20)$, where there was greater range in scores than in HS women, revealed the expected negative correlation between digit ratio and sexual orientation $(r(18)=-.30$ for sexual experience, $r$ $(18)=-.18$ for fantasy, on the left hand) but these were not significant, given the small sample size.

Previous research has examined associations between testosterone and 2D:4D. There was a significant correlation in NHS women between testosterone and right hand ratio, $r(17)=.56, p=.014$, but not the left hand ratio, $r(18)=.11, p=.655$. There was no significant correlation in HS women between testosterone and right hand ratio, $r(74)=-.10, p=.405$, or left hand ratio, $r(74)=-.04, p=.754$. 


\section{Digit ratios and spatial abilities}

No significant associations were found between spatial abilities and 2D:4D digit ratios: right hand ratio and spatial composite, $r(82)=.07, p=.533$, Paper Folding, $r(82)=.16$, $p=.159$, MRT, $r(82)=.08, p=.506$, GZSO, $r(82)=-.06$, $p=.590$; left hand ratio and spatial composite, $r(83)=.06$, $p=.619$, Paper Folding, $r(83)=-.004, p=.970$, MRT, $r(83)=.02, p=.836$, GZSO, $r(83)=.12, p=.302$.

To summarize, a significant association between sexual orientation and spatial abilities was found; NHS women performed better on the spatial tests than HS women. In contrast, neither of these variables was related to individual differences in the 2D:4D ratio.

\section{Discussion}

In the present study, NHS women exhibited better spatial performance than HS women. On an individual level, spatial ability was found to correlate with the amount of same-sex fantasy. These results are in agreement with Rahman and Wilson (2003b), but contrast with previous reports that failed to find any differences in spatial ability related to sexual orientation in women (e.g. Gladue and Bailey, 1995; Neave et al., 1999; Tuttle and Pillard, 1991). Previous studies did not control for oral contraceptive use or time of day effects, and only one controlled for menstrual cycle phase. All these variables can influence the measured level of spatial ability. Inspection of the graphs in many papers (e.g. Neave et al., 1999; Wegesin, 1998) show patterns in the expected directions that did not reach statistical significance, possibly due to uncontrolled error variation. When relevant factors were controlled, we were able to demonstrate a significant association between sexual orientation and spatial ability. The finding from the present study that NHS women performed better on spatial tasks despite no difference in circulating testosterone provides evidence consistent with the hypothesis that NHS and HS women differ in their exposure to prenatal androgens, and that prenatal androgens are related to adult spatial abilities.

The lack of any differences between HS and NHS women in the $2 \mathrm{D}: 4 \mathrm{D}$ digit ratio is in accordance with Lippa (2003), but contrary to reports suggesting a masculinized ratio in homosexual women (McFadden and Shubel, 2002, 2003; Rahman and Wilson, 2003a; Williams et al., 2000). The inconsistency between the present study and previous reports may be due to the fact that our NHS participants were not pre-selected to have a strictly lesbian sexual orientation. Our sample consisted of strictly heterosexual and non-strictly heterosexual women, who might be classified as bisexual but not lesbian. It may be that comparisons of strictly lesbian and heterosexual women are required to see digit ratio differences. In support of this, we did find a correlation of $r=-.30$ within the NHS group between the 2D:4D ratio and same-sex sexual experience.
Alternatively, a lack of correlation between sexual orientation and digit ratio might suggest that prenatal androgens alter these two characteristics at different time points during gestation.

In men, individual differences in spatial abilities have been found to correlate with the 2D:4D ratio (Manning and Taylor, 2001; McFadden and Shubel, 2003; c.f. Coolican and Peters, 2003). Although our study controlled for several known factors that affect spatial performance including time of day (Moffat and Hampson, 1996) and stage of the menstrual cycle (Hampson, 1990a), we did not find evidence for an association between digit ratios and spatial abilities in women. This supports a majority of recent studies that have not found significant correlations in women, even with large samples (Austin et al., 2002; Coolican and Peters, 2003; McFadden and Shubel, 2003; but see Csathó et al., 2003 for an exception). It is possible that the physiological fluctuations in prenatal androgens that occur in females are not sufficient to affect the digit ratios of healthy women, though the ratio may be affected in exceptional conditions such as $\mathrm{CAH}$, where androgen levels approach the male range (Brown et al., 2002; Ökten et al., 2002). Indeed, the sex difference in 2D:4D accounts for only $6 \%-9 \%$ of the total variance in digit ratios (Coolican and Peters, 2003), which presumably represents the maximum possible contribution of prenatal androgens. Alternatively, as mentioned above, it is possible that digit ratios and spatial abilities are uncorrelated in females because they differentiate under androgens during different gestational periods, and high androgens during one period do not necessarily denote high androgens during another. Correlations in males could reflect the sustained output of testicular androgens over a prolonged period of development.

The present study provides some of the first controlled evidence that increased spatial abilities and a heteroflexible sexual orientation are positively associated. This relationship is consistent with evidence that shows an association between prenatal androgens and both spatial abilities (e.g. Grimshaw et al., 1995) and lesbian sexual orientation (e.g. Brown et al., 2002a; Dittmann et al., 1992; McFadden and Pasanen, 1998; Zucker et al., 1996). However, it is possible that both increased spatial abilities and a non-heterosexual orientation are associated because of some other unidentified variable. Future studies will be needed to address this possibility. For example, participation in sports or other regular spatial activities may improve spatial processing (Baenninger and Newcombe, 1989), and it is plausible that some variable may underlie heteroflexibility and increased sports participation (e.g. sensation-seeking, bodily confidence, etc.). As well, the lack of significant correlations between digit ratios and spatial abilities or sexual orientation in the present study points to the possibility that the $2 \mathrm{D}: 4 \mathrm{D}$ ratio may not be an effective marker of androgen exposure during all periods of gestational development in healthy women. 


\section{Acknowledgments}

This article is based on S. M. van Anders' master's thesis at the University of Western Ontario. We would like to thank the editor and two anonymous reviewers for their helpful suggestions. We would also like to thank Erin Morley for doing the digit measurements for the reliability analysis. S. M. van Anders is now at Simon Fraser University. This research was supported by a grant to E. Hampson from the Natural Sciences and Engineering Research Council of Canada (NSERC). S. M. van Anders was supported by an NSERC Postgraduate Scholarship.

\section{References}

Austin, E.J., Manning, J.T., McInroy, K., Mathews, E., 2002. A preliminary investigation of the associations between personality, cognitive ability and digit ratio. Pers. Indiv. Diffs. 33, 1115-1124.

Baenninger, M., Newcombe, N., 1989. The role of experience in spatial test performance: a meta-analysis. Sex Roles. 20, 327-344.

Buck, J.J., Williams, R.M., Hughes, I.A., Acerini, C.L., 2003. In-utero androgen exposure and 2nd to 4th digit length ratio-comparisons between healthy controls and females with classical congenital adrenal hyperplasia. Hum. Reprod. 18, 976-979.

Brown, W.M., Finn, C.J., Cooke, B.M., Breedlove, S.M., 2002a. Differences in finger length ratios between self-identified "butch" and "femme" lesbians. Arch. Sex. Behav. 31, 123-127.

Brown, W.M., Hines, M., Fane, B.A., Breedlove, S.M., 2002b. Masculinized finger length patterns in human males and females with congenital adrenal hyperplasia. Horm. Behav. 42, 380-386.

Coolican, J., Peters, M., 2003. Sexual dimorphism in the 2D/4D ratio and its relationtomentalrotationperformance. Evol. Hum. Behav. 24, 179-183.

Csathó, A., Osváth, A., Karádi, K., Bicsák, É., Manning, J., Kállai, J., 2003. Spatial navigation related to the ratio of second to fourth digit length in women. Learn. Individ. Diffs. 13, 239-249.

Dittmann, R.W., Kappes, M.E., Kappes, M.H., 1992. Sexual behavior in adolescent and adult females with congenital adrenal hyperplasia. Psychoneuroendocrinology 17, 153-170.

Ehrhardt, A.A., Evers, K., Money, J., 1968. Influence of androgen and some aspects of sexually dimorphic behavior in women with the late-treated adrenogenital syndrome. Johns Hopkins Med. J. 123, 115-122.

Ekstrom, R.B., French, J.W., Harman, HH., Dermen, D., 1976. Kit of Factor-Referenced Cognitive Tests. Educational Testing Service, Princeton, J.J.

Gladue, B.A., Bailey, J.M., 1995. Spatial ability, handedness, and human sexual orientation. Psychoneuroendocrinology 20, 487-497.

Grimshaw, G.M., Sitarenios, G., Finegan, J.A., 1995. Mental rotation at 7 years: relations with prenatal testosterone levels and spatial play experiences. Brain Cogn. 29, 85-100.

Guilford, J.P., Zimmerman, W.S., 1956. Guilford-Zimmerman Aptitude Survey. Beverly Hills, Sheridan Supply Company.

Hall, L.S., Love, C.T., 2003. Finger-length ratios in female monozygotic twins discordant for sexual orientation. Arch. Sex. Behav. 32, 23-28.

Hampson, E., 1990a. Variations in sex-related cognitive abilities across the menstrual cycle. Brain. Cogn. 14, 26-43.

Hampson, E., 1990b. Estrogen-related variations in human spatial and articulatory-motor skills. Psychoneuroendocrinology 15, 97-111.

Hampson, E., Rovet, J.F., Altmann, D., 1998. Spatial reasoning in children with congenital adrenal hyperplasia due to 21-hydroxylase deficiency. Dev. Psychol. 14, 299-320.

Hausmann, M., Slabbekoorn, D., Van Goozen, S.H.M., Cohen-Kettenis, P.T., Gunturkun, O., 2000. Sex hormones affect spatial abilities during the menstrual cycle. Behav. Neurosci. 114, 1245-1250.
Hines, M., Fane, B.A., Pasterski, V.L., Mathews, G.A., Conway, G.S., Brook, C., 2003. Spatial abilities following prenatal androgen abnormality: targeting and mental rotations performance in individuals with congenital adrenal hyperplasia. Psychoneuroendocrinology 28, 1010-1026.

Lippa, R.A., 2003. Are 2D:4D finger-length ratios related to sexual orientation? Yes for men, no for women. J. Pers. Soc. Psychol. 85, $179-188$.

Manning, J.T., Taylor, R.P., 2001. 2nd to 4th digit ratio and male ability in sport: implications for sexual selection in humans. Evol. Hum. Behav. $22,61-69$.

Manning, J.T., Scutt, D., Wilson, J., Lewis-Jones, D.I., 1998. The ratio of 2nd to 4 th digit length: a predictor of sperm numbers and concentrations of testosterone, luteinizing hormone and oestrogen. Hum. Reprod. 13, $3000-3004$.

Manning, J.T., Barley, L., Walton, J., Lewis-Jones, D.I., Trivers, R.L., Singh, D., Thornhill, R., Rohde, P., Bereczkei, T., Henzi, P., Soler, M., Szwed, A., 2000. The 2nd:4th digit ratio, sexual dimorphism, population differences, and reproductive success: evidence for sexually antagonistic genes? Evol. Hum. Behav. 21, 163-183.

McFadden, D., 1998. Sex differences in the auditory system. Dev. Neuropsychol. 14, 261-298.

McFadden, D., Champlin, C.A., 2000. Comparison of auditory evoked potentials in heterosexual, homosexual, and bisexual males and females. J. Assoc. Res. Otolaryngol. 1, 89-99.

McFadden, D., Pasanen, E.G., 1998. Comparisons of the auditory systems of heterosexuals and homosexuals: click-evoked otoacoustic emissions. Proc. Natl. Acad. Sci. U. S. A. 95, 2709-2713.

McFadden, D., Shubel, E., 2002. Relative lengths of fingers and toes in human males and females. Horm. Behav. 42, 492-500.

McFadden, D., Shubel, E., 2003. The relationships between otoacoustic emissions and relative lengths of fingers and toes in humans. Horm. Behav. 43, 421-429.

Moffat, S.D., Hampson, E., 1996. A curvilinear relationship between testosterone and spatial cognition in humans: possible influence of hand preference. Psychoneuroendocrinology 21, 323-327.

Moffat, S.D., Hampson, E., Hatzipantelis, M., 1998. Navigation in a "virtual" maze: sex differences and correlation with psychometric measures of spatial ability in humans. Evol. Hum. Behav. 19, $73-87$.

Money, J., Schwartz, M., Lewis, V.G., 1984. Adult erotosexual status and fetal hormonal masculinization and demasculinization: 46,XX congenital virilizing adrenal hyperplasia and 46,XY androgen-insensitivity syndrome compared. Psychoneuroendocrinology 9, 405-414.

Neave, N., Menaged, M., Weightman, D.R., 1999. Sex differences in cognition: the role of testosterone and sexual orientation. Brain Cogn. $41,245-262$.

Ökten, A., Kalyoncu, M., Yaris, N., 2002. The ratio of second- and fourthdigit lengths and congenital adrenal hyperplasia due to 21-hydroxylase deficiency. Early Hum. Dev. 70, 47-54.

Peters, M., Laeng, B., Latham, K., Jackson, M., Zaiyouna, R., Richardson, C., 1995. A redrawn Vandenberg and Kuse mental rotations test: different versions and factors that affect performance. Brain. Cogn. 28, 39-58.

Peters, M., Mackenzie, K., Bryden, P., 2002. Finger length and distal finger extant patterns in humans. Am. J. Phys. Anthropol. 17, 209-217.

Rahman, Q., Wilson, G.D., 2003a. Large sexual-orientation-related differences in performance on mental rotation and judgment of line orientation tasks. Neuropsychology 17, 25-31.

Rahman, Q., Wilson, G.D., 2003b. Sexual orientation and the 2nd to 4th finger length ratio: evidence for organising effects of sex hormones or developmental instability? Psychoneuroendocrinology 28, 288-303.

Resnick, S.M., Berenbaum, S.A., Gottesman, I.J., Bouchard, T.J., 1986. Early hormonal influence on cognitive functioning in congenital adrenal hyperplasia. Dev. Psychol. 22, 191-198.

Tuttle, G.E., Pillard, R.C., 1991. Sexual orientation and cognitive abilities. Arch. Sex. Behav. 20, 307-318

Vandenberg, S.G., Kuse, A.R., 1978. Mental Rotations: a group test of viewer-centered spatial visualization. Percept. Mot. Skills 47, $599-604$ 
Vernon, P.E., 1971. The Structure of Human Abilities. Methuen, London. Voyer, D., Voyer, S., Bryden, M.P., 1995. Magnitude of sex differences in spatial abilities: a meta-analysis and consideration of critical variables. Psychol. Bull. 117, 250-270.

Wegesin, D.J., 1998. A neuropsychologic profile of homosexual and heterosexual men and women. Arch. Sex. Behav. 27, 91-108.
Weschler, E., 1981. The Weschler Adult Intelligence Scale-Revised. The Psychological Corporation, New York.

Williams, R.J., Pepitone, M.E., Chistensen, S.E., Cooke, B.M., Huberman, A.D., Breedlove, N.J., Breedlove, R.J., Jordan, C.L., Breedlove, S.M., 2000. Finger-length ratios and sexual orientation. Nature $404,455-456$. 